\title{
A Framework for Retargetable Code Generation using Simulated Annealing
}

\author{
Bert-Steffen Visser \\ University of Twente \\ Department of Computer Science \\ 7500 AE Enschede, The Netherlands \\ bvisser@natlab.research.philips.com
}

\begin{abstract}
Co-development of hardware and software is a methodology dealing with the increased design complexity of embedded systems. Retargetable code generation is a co-designing method to map a high-level software description onto a variety of hardware architectures without the need to rewrite a compiler. Highly efficient code generation is required to meet, for example, timing, area and low-power constraints. The traditional ordering of code generation phases introduces inefficiencies in the code generation process; phase-coupling deals with these inefficiencies. We introduce a new code generation technique based on simulated annealing. This technique focuses especially on highly irregular DSP architectures and is part of a generic framework for retargetable code generation. This approach is new because it fully tackles the phase-coupling problem. Furthermore, this approach shows that the modeling of the software algorithm and the hardware architecture plays a key role in the efficiency of code generation.
\end{abstract}

\section{Introduction}

Hardware/software codesign [1] is a methodology addressing the simultaneous development of hardware and software for a complex embedded system. The partitioning of an embedded system into software and hardware is one of its main problems. Related technical problems are, for example, co-modeling and co-simulation of software and hardware, and optimization of hardware usage for a specific software algorithm. Besides technical problems, codesign also takes economical problems like short-timeto-market and cost into account.

Retargetable code generation (RCG) [2][3] facilitates the codesign process. It makes it possible to automatically map a high-level software description (in for example $\mathrm{C} / \mathrm{C}++$ ) of an algorithm onto a number of different hardware architectures. This allows for a quick assessment of the most suitable architecture.

The retargetability level of a code generator is the effort to adapt a code generator to another hardware architecture. Figure 1 shows the model we use in our framework. This model has the highest level of retargetability because both software algorithm and hardware model are parameters for the code generator. [3] also discusses models with a lower level of retargetability.

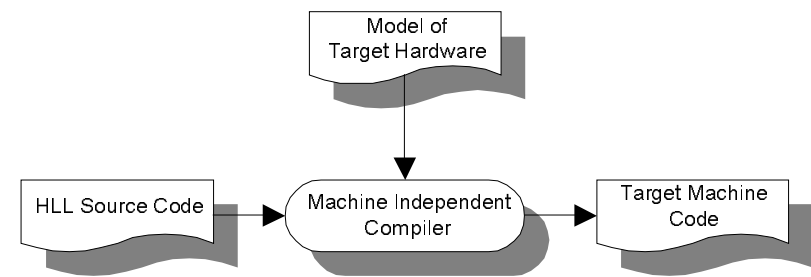

Figure 1. Machine independent compiler.

The two driving-forces behind RCG are the areas of compiler technology and high-level synthesis. Both areas differ in the treatment of the hardware model. Compiler technology uses behavioral models, while high-level synthesis uses structural models. Behavioral models describe a hardware architecture in terms of its instruction set. Its high abstraction level is a major advantage, but it makes efficient code generation for highly irregular architectures hard. Structural models describe a hardware architecture in terms of its control and data path. Consequently, its high level of detail is more suitable for the modeling of highly irregular architectures. Although, it makes code generation slow. [4] discusses a number of different behavioral and structural models.

The objectives for RCG differ from the objectives of 'traditional' code generation. Traditional compilers aim at highly regular multi-purpose processors. The hardware cannot be changed. Software is complex, large and serial. Compilation times are short. Our retargetable code generator supports highly irregular dedicated processors. Hardware can be modified. Software tends to be small and highly parallel. Compilation times can be very long.

The following hardware characteristics emphasize the irregularity of the hardware architectures.

- Pipelining (arithmetic and control).

- Irregular registers, register files and memories.

- Varying bus widths and multiple busses.

- Complex addressing mechanisms like auto-increment and modulo addressing.

- Zero-overhead loops. 
Code generation is usually separated in three successive phases [5]: instruction selection, register allocation and instruction scheduling. The disadvantage of this separation is the restriction of alternatives of one phase on the next. For example, a certain code selection limits the number of register allocation alternatives. Phase-coupling algorithms [5] cope with these phase-ordering problems.

We developed a new method based on simulated annealing to fully integrate the phases in the code generation process. Simulated annealing [6] is a generic search heuristic often applied in high-level synthesis optimization problems. It is an altered hill-climbing algorithm. This heuristic searches for neighboring solutions, given an initial solution. A fitness function, which evaluates each solution, and some probability function determines the acceptance of a solution.

This paper is structured as follows. Section 2 formulates the problem. Thereafter, section 3 discusses related work. Section 4 presents our model for an RCG framework; key components are the graph models for the intermediate representation and the hardware model (section 5). The mapping of the hardware model onto the intermediate representation is fully phase-coupled; it is highlighted in section 6. Finally, section 7 concludes this paper.

\section{Problem statement}

The level of retargetability and the generation of highly efficient code in a RCG framework are the key problems. Summarizing, the following problems can be derived.

- High-level language independence. Depending on the application domain, different specification and modeling languages are used.

- Hardware independence. The compiler should be independent of hardware specific details; a hardware model should comprise such details.

- Hardware optimizations. The compiler should be independent of hardware specific optimizations. Of course, a method to specify such optimizations should be included.

- Support of different target architectures. This constraint is evident. The hardware model allows the modeling of different hardware characteristics. Thus, a variety of architectures can be supported.

- High code quality. The generated code should be of high quality in order to meet constraints like real-time response, high dependability, execution speed and compact code (favors size).

- Reusability and extensibility. The reusability and extensibility largely depends on the model for the software algorithm and the hardware (see above).

We aim at a retargetable code generator for highly irregular architectures, especially DSP architectures. We use a structural hardware model in order to include the details for efficient code generation. Furthermore, the model can be easily extended with special hardware characteristics. We assume that the instruction set is not fixed in order to exploit the maximum level of parallelism in the data path. Our code generation algorithm supports full phase-coupling using simulated annealing. This method proves to be flexible and extensible.

\section{Related work}

RCG systems have been broadly investigated in the last few years. Examples are the Common Bus Compiler (CBC) [4], Chess [7] and Flexware [8]. These compilers target ASIP's and DSP's. The algorithms are specified in $\mathrm{C}, \mathrm{C}++$, VHDL (Flexware) or are represented in control/data flow graph representations (CBC, Chess). The hardware model in Chess and Flexware are based on mixed-level models, i.e. comprising both behavioral and structural aspects. CBC uses a behavioral model. Another interesting approach is RCG based on Integer Linear Programming (ILP) [9]; the mapping of the software onto the hardware is reformulated as an ILP-problem with many constraints. Partial phase-coupling algorithms are supported by $\mathrm{CBC}$ and ILP; register allocation and instruction scheduling are phase-coupled.

\section{Framework}

The machine independent compiler depicted in figure 1 is decomposed into a front-end and a back-end (figure 2). The front-end translates the algorithm into an intermediate representation, which is hardware independent and highlevel language independent. The back-end generates the target machine code (VLIW) and the format of the machine code. The front-end is decomposed into a syntactical and semantical analyzer. The back-end performs machine dependent optimizations, contains the phase-coupling algorithm and the instruction code generator; a monitor provides feedback of each process.

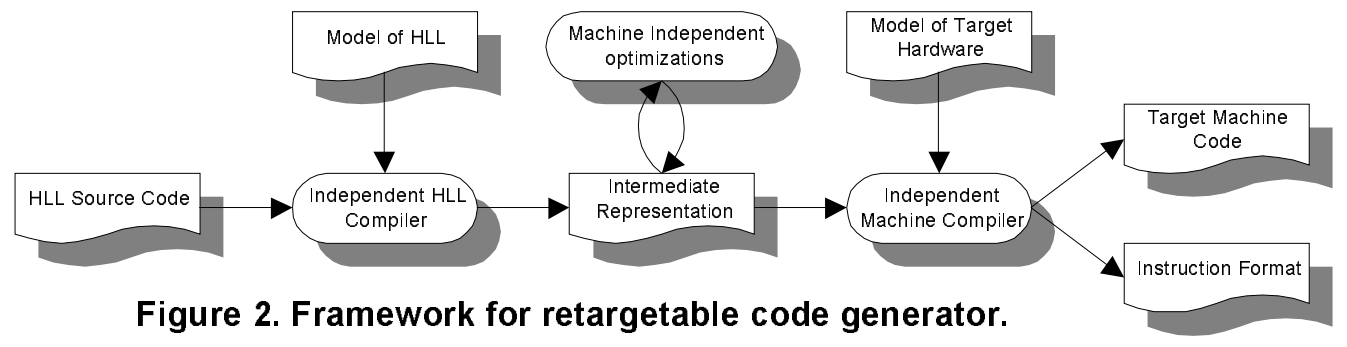


This model favors multiple input high-level languages, simply by replacing the front-end. Generally, if there are $\mathrm{N}$ input languages and $\mathrm{M}$ output languages, only $\mathrm{N}+\mathrm{M}$ frontends/back-ends are required instead of $\mathrm{N}^{*} \mathrm{M}$ compilers.

Machine independent optimizations like e.g. algebraic optimizations, constant propagation and dead-code elimination can be performed on the intermediate representation of the algorithm. Machine dependent optimizations are a separate module in the back-end and can be replaced or extended at wish.

We will now focus on the hardware model, the intermediate representation and the phase-coupling algorithm.

\section{Representation models}

\subsection{Hardware model}

The hardware model is a structural model and is based on an extended directed graph. It is derived from the data path. This representation best suits our purposes, because it models the irregularity of the hardware architecture best, including different bit widths. Three types of nodes are distinguished.

- Functional nodes denote the behavior of the data path. It models functional units like ALU's and the controller, which is considered to be a black box.

- Storage nodes denote the state within the data path. It models registers, register files and data memory.

- Connection nodes denote the structure of the data path. It models complex busses with associated multiplexers and/or tri-state drivers. Single busses (one-to-one connections between functional and storage nodes) are modeled as edges.

Nodes are annotated by a bit size, a delay time and pipelining capabilities. Delay time and pipelining are not applicable for connection nodes.

Multi-nodes introduce hierarchy; it allows the grouping of a set of functional nodes or storage nodes. For example, an ALU with different functions but the same interface is modeled as a functional multi-node; likewise a register file or data memory is modeled as a storage multi-node.

Figure 3 illustrates the graph model. It depicts a part of a DSP architecture: a main bus with a data memory, a register file, two ALU's and a shifter. All nodes and connections are 16 bits, unless annotated otherwise. Operations in an ALU can have different pipelining capabilities, e.g. in 'ALU A' the 'and' and 'or' have a pipeline of 1, the 'add' and 'sub' have a pipeline of 2 and the 'pass' has a zero delay.

\subsection{Intermediate representation}

The intermediate representation is based on a control/data flow graph (CDFG); it is an altered SIL graph [10][11]. It has a broad class of node types: constant,

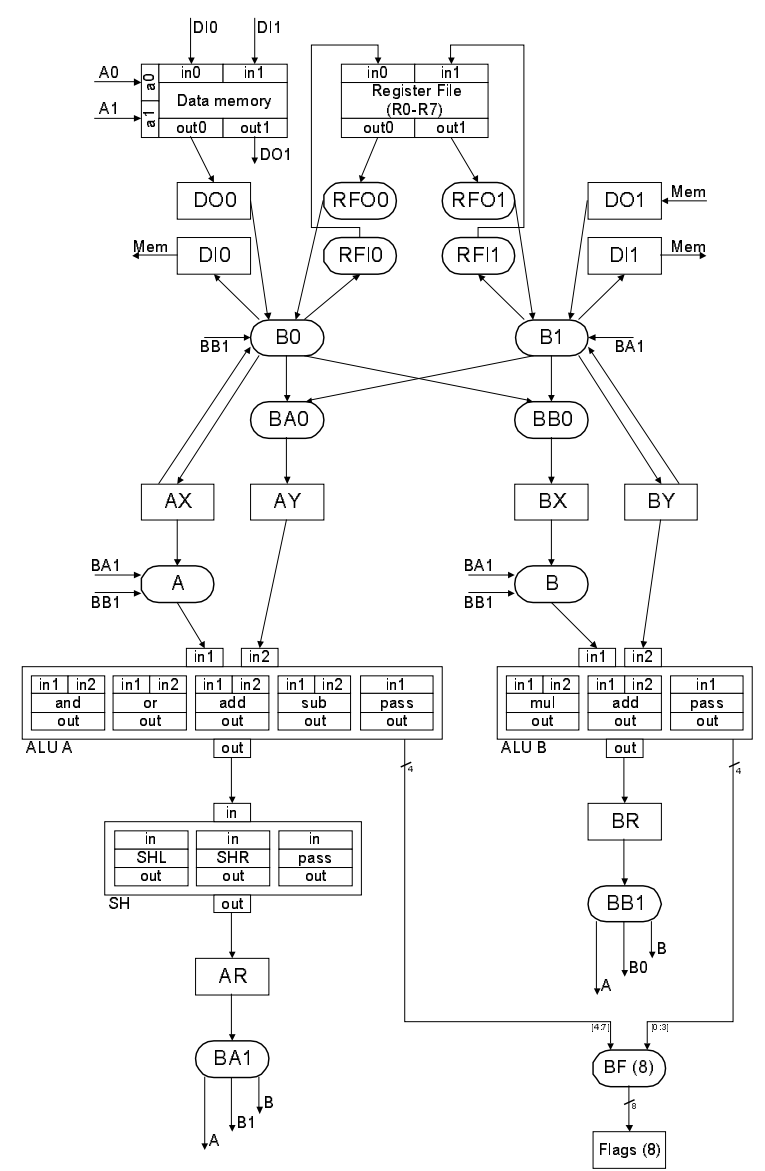

Figure 3. Example hardware graph.

operator, conditional, I/O, hierarchical, array object, store and fetch nodes. Control/data flow edges represent the flow of the algorithm; sequence edges enforce a partial ordering between the nodes. Figure 5 and 6 illustrate very simple CDFG's.

A CDFG allows the expression of fine-grained parallelism in the algorithm. The combination of control and data into a single graph clearly shows the dependencies between control and data. Furthermore, a broad number of algebraic, control and data flow optimizations are defined for CDFG's; these optimizations are machine independent (see figure 2).

\section{Phase-coupling algorithm}

The phase-coupling algorithm comprises a two-layer algorithm; figure 4 depicts the algorithm. The top-layer is based on a simulated annealing algorithm and the bottom layer is a scheduling and allocation heuristic.

The top-layer globally schedules and allocates the resources of the hardware model onto the intermediate representation (initial solution). It only uses simple hardware information including the functional units, storage elements and the interconnection scheme. Furthermore, it generates neighboring solutions by slightly changing a schedule or allocation. 


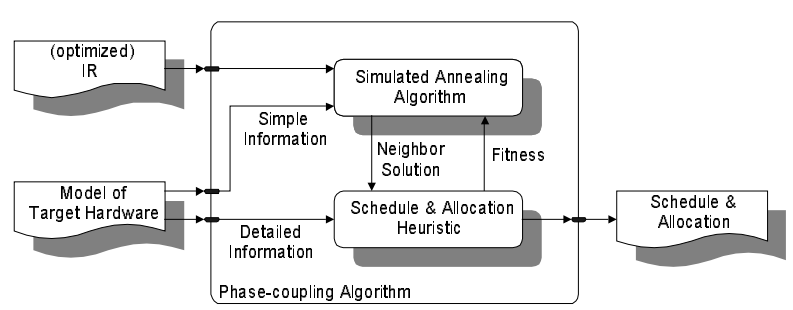

Figure 4. Decomposition of algorithm.

The bottom-layer computes a fitness value for a neighbor solution using detailed hardware information including timing constraints and pipelining capabilities. In turn, the computed fitness value is used by the top-layer to determine whether the new neighbor solution is accepted.

We will now focus on the key issues of the simulated annealing algorithm: the representation of a solution, the initial solutions and the neighbor solutions. For brevity, we will not discuss the fitness computation; obvious choices are for example the minimization of total execution time or total amount of allocated registers.

\subsection{Representation}

Annotation of the intermediate representation (IR) by schedule and allocation information from the hardware model (HM) represents a solution. Allocations are represented by the following annotations.

- Operations. HM functional nodes are allocated onto IR operator nodes. Clearly, the operation of both nodes has to match. This process equals code selection.

- Operands. HM storage or connection nodes directly connected to HM functional nodes model the operands. The input/output ports of an IR operator node are annotated with these storage nodes.

- Data transfers. A data transfer in the HM relocates data from a source storage node to a destination storage node. The interconnection scheme determines the number of possible paths. The paths are enumerated; one path is assigned to an IR control/data flow edge.

Annotations with natural numbers represent the schedules.

- Operations. A time stamp defines the ordering relation between operations. Operations can only have equal numbers if they are not allocated onto the same HM functional node. Furthermore, IR sequence edges limit the number of possibilities.

- Operands. Data in operands need to be available before an operation starts. The schedule and allocation heuristic addresses this.

- Data transfers. Data transfers conflict if two transfers share a common path. An annotated priority stamp defines the precedence in case of conflicts.

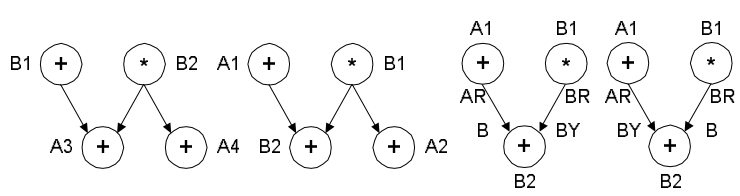

Figure 5. Example initial solutions.

\subsection{Initial solutions}

An initial solution already provides a complete code selection, register allocation and instruction schedule, but probably is highly inefficient. Operations are allocated randomly chosen matching $\mathrm{HM}$ functional nodes; they are scheduled using the list scheduling technique [5]. Thereafter, operands matching with the operations are selected. Finally, a randomly chosen data transfer selected from the enumerated list is assigned. Depending on the life time and the arrival time (both determined by the operation's schedule) the data transfers are assigned priorities.

Figure 5 shows example initial solutions for operations (left) and operands (right); 'ALU A' is denoted by 'A', 'ALU B' is denoted by B. The hardware model in figure 3 is used.

Possible data transfers from $A R \rightarrow B$ are listed below. Long data transfers introduce spill code, which is used to reduce bus loads.

- $\mathrm{AR} \rightarrow \mathrm{BA} 1 \rightarrow \mathrm{B}$.

- $\mathrm{AR} \rightarrow \mathrm{BA} 1 \rightarrow \mathrm{B} 1 \rightarrow \mathrm{BB} 0 \rightarrow \mathrm{BX} \rightarrow \mathrm{B}$.

- $\mathrm{AR} \rightarrow \mathrm{BA} 1 \rightarrow \mathrm{B} 1 \rightarrow \mathrm{RFI} 1 \rightarrow \mathrm{R} 4 \rightarrow \mathrm{RFO} 1 \rightarrow \mathrm{B} 1 \rightarrow \mathrm{BB} 0 \rightarrow \mathrm{B}$ $\mathrm{X} \rightarrow \mathrm{B}$.

\subsection{Neighbor solutions}

A neighbor solution is a slightly changed schedule or allocation for an operand, operation or data transfer. The occurrence of such a transformation is parameterizable by the following probabilities (PS is probability for schedule, PA is probability for allocation). E.g. the probability on an operation schedule transformation is: $\mathrm{P}_{\text {operation }} \cdot \mathrm{PS}_{\text {operation. }}$

- $\mathrm{P}_{\text {operation }}+\mathrm{P}_{\text {operand }}+\mathrm{P}_{\text {datatransfer }}=1$.

- $\mathrm{PS}_{\text {operation }}+\mathrm{PA}_{\text {operation }}=1$.

- $\mathrm{PS}_{\text {datatransfer }}+\mathrm{PA}_{\text {datatransfer }}=1$.

Figure 6 shows four different transformations to illustrate the generation of neighbor solutions. ' $A$ ' and ' $B$ ' denote the ALU's in figure 3. The numbers represent the schedule/priority stamps. Other letter combinations represent HM storage or connection nodes.

(A) Reallocation of operations. The top left node is reassigned to 'ALU B'. The resulting conflict on schedule 1 - ALU B' is used twice - is resolved by increasing the schedule step of the top right node and propagating the effect through the rest of the graph.

(B) Rescheduling of operations. Operations having no ordering relation can be rescheduled; operations that are not on the same directed path are not related. The schedule of the top left and the bottom right are exchanged. 


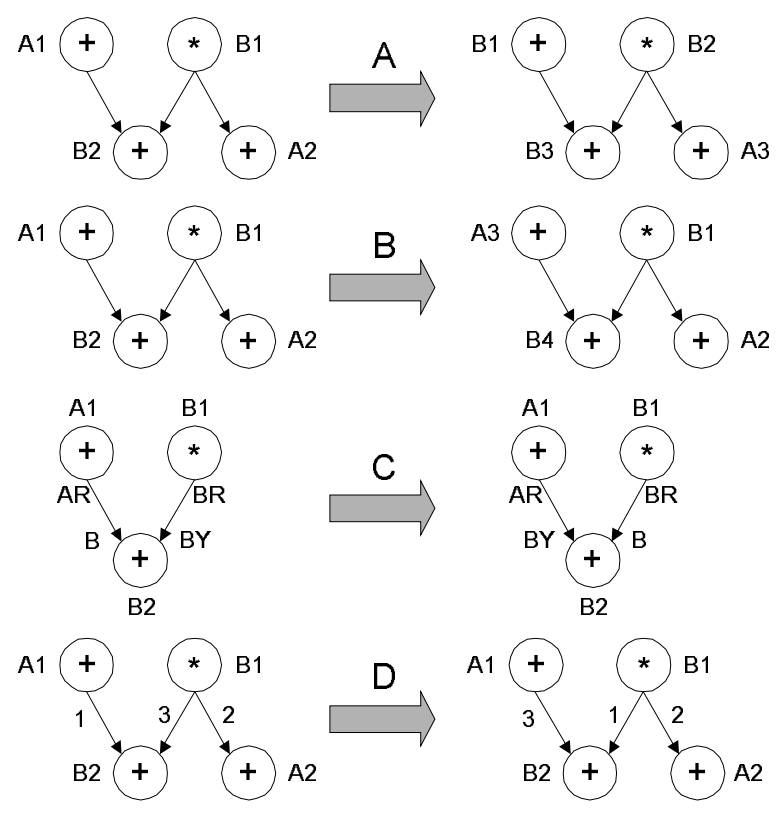

Figure 6. Finding neighbor solutions.

(C) Reallocation of operands. The ' $\mathrm{B}$ ' and ' $\mathrm{BY}$ ' are exchanged. This also results in different data transfers.

(D) Rescheduling of data transfers. The priority of the left two edges is exchanged.

The last case, the reallocation of data transfers, is not separately shown; for a randomly selected edge another data transfer is selected from the enumerated list.

After the transformation, the scheduling and allocation heuristic computes a fitness value. Depending on the probability function in the simulated annealing algorithm, the neighbor solution is accepted or rejected. Whenever the stopping condition is reached, the algorithm terminates.

\section{Conclusions}

The key problems for the definition of an extensible RCG framework are the modeling of the software algorithm, the modeling of the target hardware and finding an efficient mapping from the software to the hardware, while bearing in mind the hardware is highly irregular. The intermediate representation, describing the algorithm, supports fine-grained parallelism and a large number of optimizations; it also meets the requirement of high-level language independence. The hardware model is very flexible and extensible, meeting the requirement of hardware independence. Finally, the fully phase-coupled code generation algorithm allows a seamless mapping of the software onto the hardware. The fitness function models the design goals and can easily be adjusted.

The incorporation of the compact code constraint in the fitness function is still a topic for further research. Our assumption to use a non-fixed instruction set causes this problem, because it is now hard to control the code size. Possibly, a scheme of removing unused or little used parallelism can contribute to the compact code constraint. Furthermore, code compaction techniques, like Huffman encoding, can be thought of.

We used a simple prototype to verify our approach. We actually mapped a simple data flow algorithm onto the hardware model in figure 3 , while minimizing on the number of registers and memory locations. We performed 10 test runs and in all runs the optimal result of 19 locations could be found. The convergence of the simulated annealing algorithm proved to be good, but of course highly depends on the complexity of the to-bemapped-algorithm, hardware description and, even more important, the parameters of the simulated annealing algorithm.

\section{Acknowledgements}

This research is part of the Transformational Design project within the Embedded Systems group of the Department of Computer Science, University of Twente.

\section{References}

[1] G. de Micheli, H. Sami, Hardware Software Codesign, Kluwer Academic Publishers, 1996.

[2] B.S. Visser, A Framework for Retargetable Code Generation based on Simulated Annealing, M.Sc. thesis, University of Twente, November 1997.

[3] P. Marwedel, Code Generation for For Embedded Processs: An Introduction. In P. Marwede1, G. Goossens (Eds.), Code Generation for Embedded Processors, Kluwer Academic Publishers, 1995, pp. 14-31.

[4] Andreas Fauth, Beyond Tool-specific Machine Descriptions. In P. Marwedel, G. Goossens (Eds.), Code Generation for Embedded Processors, Kluwer Academic Publishers, 1995, pp. 138-152.

[5] S. Bashford, Code Generation Techniques for Irregular Architectures, Report No. 596, University of Dordmund, November 1995.

[6] S. Kirkpatrich, C.D. Gelatt, M.P. Vecchi, Optimization by Simulated Annealing. Science, vol. 220, 1983, pp. 671-680.

[7] D. Lanneer, J. Van Praet, A. Kifli, K. Schoofs, W. Geurts, F. Thoen, G. Goossens, Chess: Retargetable Code Generation for Embedded DSP Processors. In P. Marwedel, G. Goossens (Eds.), Code Generation for Embedded Processors, Kluwer Academic Publishers, 1995, pp. 85-102.

[8] P.G. Paulin, C. Liem, T.C. May, S. Sutarwala, FlexWare: $A$ Flexible Firmware Development Environment for Embedded Systems. In P. Marwede1, G. Goossens (Eds.), Code Generation for Embedded Processors, Kluwer Academic Publishers, 1995, pp. 6784.

[9] T. Wilson, G.Grewal, S. Henshall, D. Banerji, An ILP-based Approach to Code Generation. In P. Marwedel, G. Goossens (Eds.), Code Generation for Embedded Processors, Kluwer Academic Publishers, 1995, pp. 103-118.

[10] W.E.H. Kloosterhuis, M.M.R. Eyckmans, J.Hofstede, C. Huijs, T. Krol, O.P. McArdle, W.J.M. Smits, L.G.L. Svensson SIL-1 Language Report, SPRITE deliverable L.S.a.a/Philips/Y3-M12/2, 1992.

[11] P.F.A. Middelhoek, Transformational Design: An Architecture Independent Interactive Design Methodology for the Synthesis of correct and efficient Digital Systems, Ph.D. thesis, University of Twente, 1997. 